\title{
Orthogonal Poly-phase Code Design Based on Adaptive Mix Algorithm in MIMO Radar Yi-rong $\mathrm{Li}^{1, a}$, Hanying $\mathrm{Hu}^{1, a}$
}

${ }^{1}$ Zhengzhou Institute of Information science and technology, Zhengzhou 450002, China

aliyirong131@163.com

\begin{abstract}
Keywords: MIMO Radar; Orthogonal Poly-phase Code; Walsh Matrix; Adaptive; Doppler
\end{abstract} Tolerance.

\begin{abstract}
A new method of orthogonal poly-phase code design is proposed to improve signal orthogonality and Doppler tolerance for MIMO radar system. First, eight-phase code which has a better compromise of freedom and processing complexity is generated by Walsh matrix. Initial code sequences are chosen from the constructed codes. Then an adaptive simulated annealing genetic mix algorithm is proposed to numerically optimize orthogonal poly-phase coded signals. Signal orthogonality and Doppler tolerance are taken into account in objective function simultaneously, cross and mutation probability is changed adaptively by optimizing progress. Simulation results show this method can design poly-phase code with lower autocorrelation side-lobe peak and superior Doppler tolerance.
\end{abstract}

\section{Introduction}

Multiple-input multiple-output(MIMO) radar has been widely researched as a modern radar system in recent years, while orthogonal waveform design is a key technology which is different from phased-array radar[1-3]. A well-designed orthogonal waveform is useful to extract independent target information so as to improve the detection property and parameter identifiability[4-5]. In this paper, we will study the technology of MIMO radar waveform design starting with orthogonal poly-phase code.

Simulated annealing(SA) algorithm[6], genetic algorithm(GA)[7] and particle swarm optimization algorithm with Hamming scan[8] are used to get poly-phase code sequences with good aperiodic autocorrelation and cross-correlation properties. However, all these algorithms use the random code sequences that will cause limitation to orthogonal performance. A method of polyphase code design based on Walsh matrix is proposed in[9]. It uses the orthogonality of Walsh code to generate initial poly-phase code, then got final codes by GA. The designed signals has low crosscorrelation and autocorrelation sidelobes. But this method only gives construction method of fourphase code which is hard to make full use of phase freedom to gain performance enhancements. Simultaneously, Doppler tolerance was ignored in all the above methods.

To overcome the problems mentioned above, a poly-phase code design method based on adaptive simulated annealing genetic mix algorithm(SAGA) is proposed in this paper. First, we extend the results in[9] to structure initial eight-phase code. Then we use adaptive SAGA to optimize initial codes. Crossover and mutation probabilities is changed adaptively to get a better optimal ability. Doppler tolerance is taken into consideration simultaneously.

\section{Signal model}

Consider a MIMO radar system transmit $L$ orthogonal poly-phase code waveforms simultaneously with a code length of $N$, the signal set ${ }^{S}$ can expressed as follows:

$$
\boldsymbol{S}=\left\{s_{l}(t)=a(t) e^{j \varphi_{l}(n)}, n=1,2, \cdots, N, \quad l=1,2, \cdots, L\right\}
$$

Assuming ${ }^{a(t)}$ is a rectangular pulse, taking consideration of the presence of Doppler effect, an 
intra-pulse phase shift ${ }^{\varphi(n)}$ can be expressed as:

$$
\varphi(n)=2 \pi f_{d}(n-1) \frac{T_{p}}{N-1}, \quad n=1,2, \cdots, N
$$

Where $T_{p}$ denotes pulse width, $f_{d}$ is Doppler shift, the aperiodic correlation function between $^{s_{p}(n)}$ and ${ }^{s_{q}(n)}$ is shown in(3). If $p=q$ (3) gives autocorrelation function, otherwise, (3) represents cross-correlation function.

$$
C R\left(\phi_{p}, \phi_{q}, f_{d}, k\right)=\left\{\begin{array}{l}
\frac{1}{N} \sum_{n=1}^{N-k} e^{j\left[\phi_{p}(n)-\phi_{q}(n+k)-\varphi(n+k)\right]}, 0 \leq k<N \\
\frac{1}{N} \sum_{n=-k+1}^{N} e^{j\left[\phi_{p}(n)-\phi_{q}(n+k)-\varphi(n+k)\right]},-N<k<0
\end{array}\right.
$$

\section{Orthogonal poly-phase code design based on adaptive SAGA algorithm}

\section{Construction of orthogonal poly-phase code based on Walsh matrix}

Assuming $\boldsymbol{A}$ is a Walsh matrix of $N \times N$, but $\boldsymbol{A}$ can only satisfy the strict orthogonality of twophase codes. [9] gives construction method of four-phase code. The increase of phase number can raise phase freedom. So we will extend phase number to eight firstly, and give a proof of it.The $2 N \times 2 N$ Walsh matrix for four-phase code given in[9] is:

$$
\boldsymbol{C}=\left[\begin{array}{cc}
\boldsymbol{A} & j \boldsymbol{A} \\
-j \boldsymbol{A} & \boldsymbol{A}
\end{array}\right]
$$

One construction of eight-phase code by Walsh matrix can be written as:

$$
\boldsymbol{B}=\left[\begin{array}{cc}
\boldsymbol{C} & -\boldsymbol{C}+j \boldsymbol{C} \\
\boldsymbol{C}-j \boldsymbol{C} & \boldsymbol{C}
\end{array}\right]
$$

where $\boldsymbol{B}$ is $4 N \times 4 N$ orthogonal matrix with the phase number of eight. The proof is shown as follows: define $\boldsymbol{b}_{i}, \boldsymbol{b}_{j}$ is the ith and jth row of $\boldsymbol{B}, \boldsymbol{c}_{i}, \boldsymbol{c}_{j}$ is the ith and jth row of $\boldsymbol{C}$, the elements is $\left(c_{i 1}, c_{i 2}, \cdots, c_{i 2 N}\right)$ and $\left(c_{j 1}, c_{j 2}, \cdots, c_{j 2 N}\right), \quad c_{i} \otimes c_{j}=0(i \neq j), \otimes$ represents vector inner product. Three conditions are considered:

First condition: ${ }^{i}$ is in the first $2 N$ rows, while $j$ is in the last $2 N$ rows, then:

$$
\begin{gathered}
\boldsymbol{b}_{i} \otimes \boldsymbol{b}_{j}=\left(c_{i 1}, c_{i 2}, \cdots, c_{i 2 N}\right) \otimes \\
\left(c_{j 1}-j c_{j 1}, c_{j 2}-j c_{j 2}, \cdots, c_{j 2 N}-j c_{j 2 N}\right)+\left(c_{j 1}, c_{j 2}, \cdots, c_{j 2 N}\right) \\
\left(-c_{i 1}+j c_{i 1},-c_{i 2}+j c_{i 2}, \cdots,-c_{i 2 N}+j c_{i 2 N}\right)
\end{gathered}
$$

Second condition: both of $i$ and $j$ are in the first $2 N$ rows, then:

$$
\begin{aligned}
\boldsymbol{b}_{i} \otimes \boldsymbol{b}_{j} & =\left(c_{i 1}, c_{i 2}, \cdots, c_{i 2 N}\right) \otimes\left(c_{j 1}, c_{j 2}, \cdots, c_{j 2 N}\right)+ \\
& \left(j c_{i 1}-c_{i 1}, j c_{i 2}-c_{i 2}, \cdots, j c_{i 2 N}-c_{i 2 N}\right) \otimes \\
& \left(j c_{j 1}-c_{j 1}, j c_{j 2}-c_{j 2}, \cdots, j c_{j 2 N}-c_{j 2 N}\right)=0
\end{aligned}
$$

Third condition: both of $i$ and $j$ are in the first rows, the proof is the same as condition two.16phase and 32-phase code can be done in the same method, that will not be covered here. The increase of phase number can improve orthogonal property, but processing complexity is raised in the contrary. Eight-phase code can get a better compromise between freedom and complexity, so our research will focus on eight-phase code.We can choose initial code sequence from the orthogonal matrix sets after elementary rank transformation, then use adaptive SAGA to optimize poly-phase signal deeply. 


\section{Poly- phase code optimization based on adaptive SAGA}

An adaptive SAGA is proposed to optimize poly-phase signal. Comparing with the constant crossover and mutation probabilities in conventional genetic algorithm, we can adaptively change crossover and mutation probabilities based on optimize progress: Use larger crossover and mutation probabilities to search quickly at the early stage of the evolution. While evolutional increases, crossover and mutation probabilities will be reduced gradually to refine precise search and reach optimal value.

The cost function contains orthogonality and Doppler tolerance is defined as:

$$
F(\Phi)=\operatorname{PSL}(\boldsymbol{C}(\boldsymbol{\Phi}, 0))+\sum_{f_{d} \neq 0}\left(\lambda_{1} P S L\left(\boldsymbol{C}\left(\boldsymbol{\Phi}, f_{d}\right)\right)+\lambda_{2} P S L\left(\boldsymbol{C}\left(\boldsymbol{\Phi},-f_{d}\right)\right)\right)
$$

Where $\quad \boldsymbol{C}\left(\boldsymbol{\Phi}, f_{d}\right)=\frac{1}{L} \sum_{p=1}^{L}\left(\left|C R\left(\phi_{p}, \phi_{p}, f_{d}, k\right)\right|+\mu_{p} \sum_{q=1, q \neq p}^{L}\left|C R\left(\phi_{p}, \phi_{q}, f_{d}, k\right)\right|\right)$

The peak sidelobe level(PSL) is defined as:

$$
\operatorname{PSL}\left(\boldsymbol{C}\left(\boldsymbol{\Phi}, f_{d}\right)\right)=\max \left(\boldsymbol{C}\left(\boldsymbol{\Phi}, f_{d}\right)_{k \neq 0}\right) / \boldsymbol{C}\left(\boldsymbol{\Phi}, f_{d}\right)_{k=0}
$$

The algorithm procedure is presented in Fig.1.

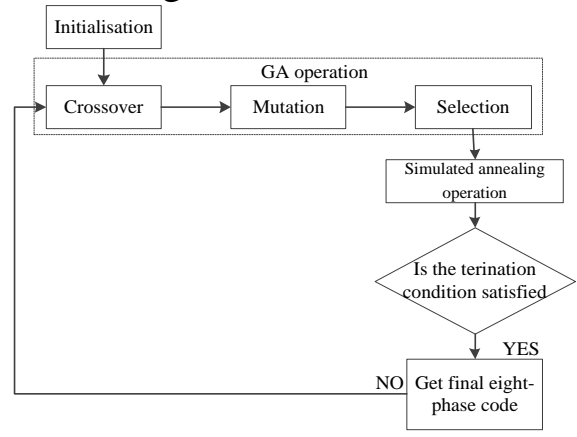

Fig.1: Algorithm procedure

Step1: (Initialisation) Constructing initial eight-phase code by 3.2, and coding it as initial population. Initial temperature is $T_{0}$, individual in population is $K$, evolutional generation is $T$, then the initial population can be represented as:

$$
\Omega_{0}=\left\{\Phi_{k}\right\} \quad k=1, \cdots, K
$$

Step2: (GA operation) To prevent prematurity and improve optimization ability, we can adaptively change crossover probability and mutation probability based on optimize progress.

$$
\begin{aligned}
& P_{c}=\left\{\begin{array}{cc}
p_{c 0} \frac{F_{\text {max }}-F_{a v}}{F_{\text {max }}-F^{\prime}} & F^{\prime} \leq F_{a v} \\
p_{c 0} & F^{\prime}>F_{a v}
\end{array}\right. \\
& P_{m}=\left\{\begin{array}{cc}
p_{m 0} \frac{F_{\text {max }}-F_{a v}}{F_{\text {max }}-F} & F \leq F_{a v} \\
p_{m 0} & F>F_{a v}
\end{array}\right.
\end{aligned}
$$

where $p_{c 0} / p_{m 0}$ is initial crossover probability/mutation probability. $F_{\max } / F_{a v}$ is the max/mean fitness value of current population. $F$ 'is the bigger fitness value of the two individuals to cross, $F$ is the fitness value of the individual to mutate.

Step3: (Simulated annealing operation) In the new population, new individual $\Phi_{k}^{\prime}$ is accepted depending on the probability:

$$
P\left(\Phi_{k}^{\prime} \rightarrow \Phi_{k}\right)=\left\{\begin{array}{cc}
\exp \left(-\frac{\Delta F}{T_{c}}\right) & \Delta F>0 \\
1 & \Delta F \leq 0
\end{array}\right.
$$


where $\Delta F=F\left(\Phi_{k}^{\prime}\right)-F\left(\Phi_{k}\right)$ represents the variation of fitness value, $T_{c}=\lambda^{t-1} T_{0}$ is the annealing temperature, $\lambda$ is a constant between 0 and $1,{ }^{t(1 \leq t \leq T)}$ is current generation.

Step4: Determine whether the termination condition is satisfied, if not ,repeat step2 and step3.

Simulation results

Simulation parameters is shown in Table 1.

Table 1: Parameters setup

\begin{tabular}{|c|c|c|c|c|c|c|c|}
\hline generation $T$ & 1000 & weight $\mu_{p}$ & 1 & population size $K$ & 50 & $\begin{array}{l}\text { Signal } \\
\text { number } L\end{array}$ & 4 \\
\hline weight $\lambda_{1}$ & 1 & weight $\lambda_{2}$ & 1 & phase number $M$ & 8 & weight $\lambda$ & 0.8 \\
\hline
\end{tabular}

Notation: $T_{0}, p_{c 0}$ and $p_{m 0}$ must adjust to practical issue to get a better result.

\section{Correlation performance}

First we do a research of short code sequence with code length $N=32$ (the length must be integer power of 2), Table 2 and Table 3 give autocorrelation sidelobe peak(ASP) and crosscorrelation peak(CP) for our algorithm and the algorithm in [9] with the Doppler shift equals to 0 respectively. We can see that our algorithm can design eight-phase code with an improvement of ASP value with $0.23-0.87 \mathrm{~dB}$ than literature[9], while mean $\mathrm{CP}$ value remain the same.

When the signal length become larger, the signal orthogonality will be improved. See Fig.2 and Fig.3 in the length of 512.The ASP value is below $-25 \mathrm{~dB}$ and CP value is below $-22 \mathrm{~dB}$, the correlation property is much better than the condition of $N=32$.

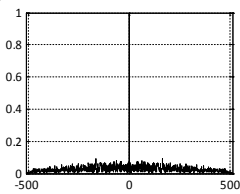

Code 1

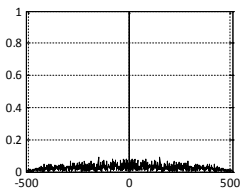

code 3

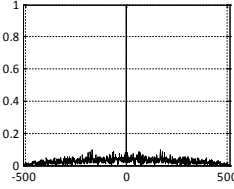

0
Code 2

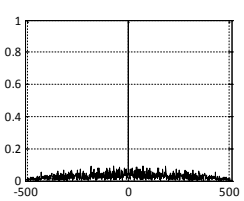

code 4

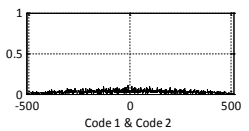

Code $1 \&$ code 2

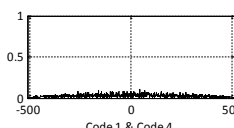

Code $1 \&$ Code 4

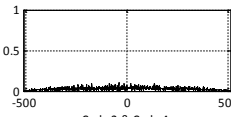

Code 2 \& Code 4
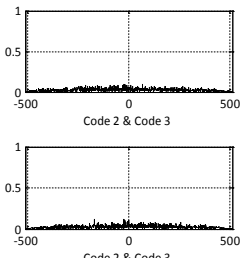

Code 2 \& Code 3

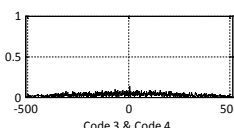

code $3 \&$ code 4

Fig.2: Autocorrelation curve of our code

Fig.3: Cross-correlation curve of our code

Table 2: Correlation peak of the eight-phase code designed by our algorithm

\begin{tabular}{lcccc}
\hline dB & Code1 & Code 2 & Code 3 & Code 4 \\
\hline Code 1 & -17.3596 & -15.4137 & -14.8531 & -15.0017 \\
Code 2 & -15.4137 & -17.3312 & -15.0479 & -15.9114 \\
Code 3 & -14.8531 & -15.0479 & -17.6313 & -15.8802 \\
Code 4 & -15.0017 & -15.9114 & -15.8802 & -17.4361 \\
\hline \multicolumn{5}{c}{ Table 3: Correlation peak of the eight-phase } \\
\hline dB code designed by literature[9] \\
\hline Code 1 & Code 1 & Code 2 & Code 3 & Code 4 \\
Code 2 & -17.1350 & -15.5651 & -15.5024 & -15.7520 \\
Code 3 & -15.5651 & -16.4653 & -15.4075 & -15.5761 \\
Code 4 & -15.5024 & -15.4075 & -17.0542 & -15.3794 \\
\hline
\end{tabular}

\section{Doppler tolerance}

Fig.4 shows the PSL changing trends with max Doppler shift $(N=32)$. The PSL of our code(named New code in the Figure) is below the code in[9] under all Doppler shifts, and is improved with 2.5-3dB.The PSL variation is less than $0.9 \mathrm{~dB}$ while the code in[9] changed about 2dB. Doppler loss is shown in Fig.5.The amplitude of code in[9] decrease rapidly with the increase of Doppler shift, the amplitude remains below -12dB after a relative Doppler shift of 0.8. While our code can enjoy slow deterioration at the same level and maintain around -10dB, decrease in the 
Doppler shift between 1.3 and 1.5,but it can also remain higher than -12dB,i.e., our algorithm can get better Doppler tolerance than[9] in a short code length.

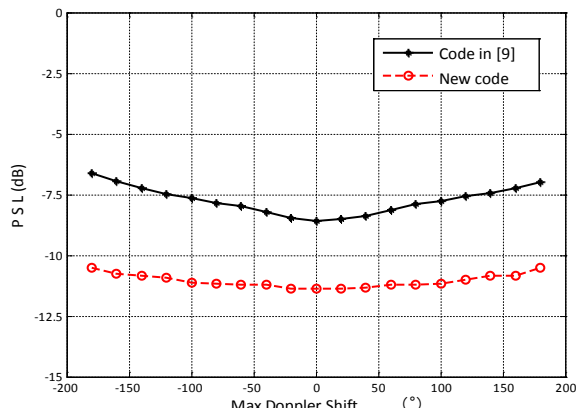

Fig.4: PSL values versus maximal Doppler shift

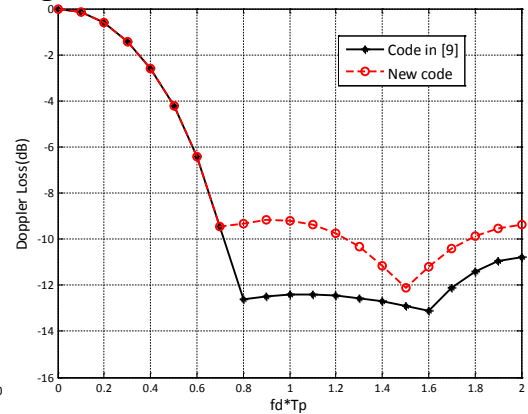

Fig.5: Doppler Loss with Doppler shift

Fig. 6 shows the PSL of $N=512$.The PSL of our code is improved with 6.4-7.3dB.The PSL variation is larger than $N=32$, but can maintain less than $1.79 \mathrm{~dB}$, while the code in[9] changed about 3.87dB. The Doppler loss is shown in Fig.7. Doppler tolerance become worse under long code length. But our code remains higher than the code in[9] with $\left|f_{d} T_{p}\right| \geq 0.8$ and maintain around $14 \mathrm{~dB}$,i.e.,our code can achieve better Doppler tolerance than code in[9] in long code length.

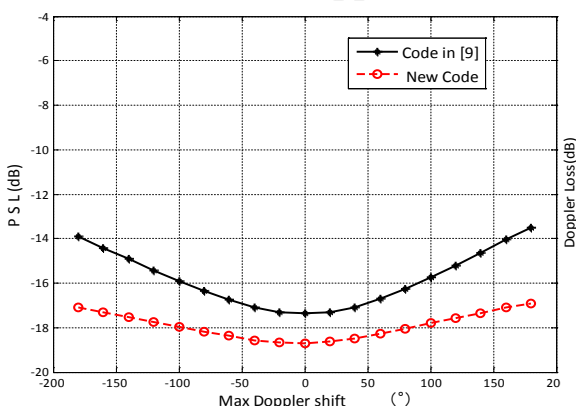

Fig.6 PSL values versus maximal Doppler shift

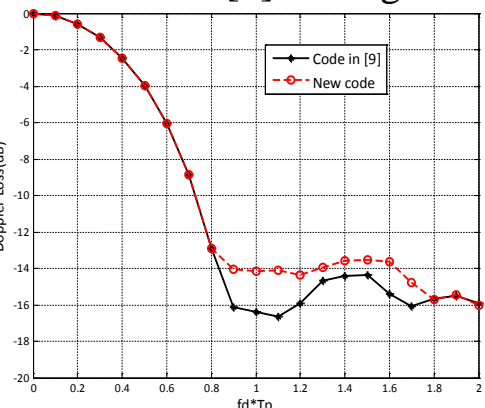

Fig.7 Doppler Loss with Doppler shift

\section{Conclusions}

This paper presented a new method of poly-phase code design based on adaptive SAGA. According to the simulation results of different code length, our code can get better orthogonality and Doppler tolerance performance.

\section{Acknowledgements}

The research work was supported by National Natural Science Foundation of China under Grant No. 61065009 and Natural Science Foundation of Qinghai Provincial under Grant No. 2011-z-756.

\section{References}

[1] Hassanien A, Vorobyov S A. Transmit energy focusing for DOA estimation in MIMO radar with colocated antennas[J]. IEEE Transactions on Signal Processing, 2011, 59(6): 2669-2682.

[2] Hua G, Abeysekera S S. MIMO radar transmit beampattern design with ripple and transition band control[J]. IEEE Transactions on Signal Processing, 2013, 61(11): 2963-2974.

[3] Hassanien A, Vorobyov S A. Phased-MIMO radar: A tradeoff between phased-array and MIMO radars[J]. IEEE Transactions on Signal Processing, 2010, 58(6): 3137-3151.

[4] Babur G, Krasnov O A, Yarovoy A, et al. Nearly Orthogonal Waveforms for MIMO FMCW Radar[J]. Aerospace and Electronic Systems,IEEE Transactions on, 2013, 49(3): 1426-1437.

[5] Song X, Willett P, Zhou S. Detection performance for statistical MIMO radar with identical and orthogonal waveforms[C]// IEEE Radar Conference (RADAR), 2011:022-026.

[6] Deng H. Polyphase code design for orthogonal netted radar systems[J]. Signal Processing, IEEE Transactions on, 2004, 52(11): 3126-3135. 
[7] Liu B, He Z, Zeng J, et al. Polyphase orthogonal code design for MIMO radar systems[C]//Radar, 2006. CIE'06. International Conference on. IEEE, 2006: 1-4.

[8] B.Roja Reddy, M.Uttara Kumari.Polyphase Orthogonal Waveform using Modified Particle Swarm Optimization Algorithm for MIMO Radar[C].IEEE International Conference on Signal Processing, Computing and Control(ISPCC),2012,3:1-6.

[9] Li Jun, Liu Na, Liu Hong-ming.A method of poly-phase code sequence design based on Walsh matrix[J].Chinese Journal of Radio Science, 2013, 28(3):577-583.

[10] Khan H A, Edwards D J. Doppler problems in orthogonal MIMOradars[C]//Radar, 2006 IEEE Conference on. IEEE, 2006: 4:244-247. 